\title{
Handekzeme: Ätiologie, Diagnostik und therapeutisches Management
}

Vera Baur, Erwin S. Schultz



\begin{abstract}
Das Handekzem ist eine multifaktorielle Hauterkrankung. Eine genetische Prädisposition, die spezifische Exposition und insbesondere berufliche Aspekte sollten im Management der Patienten berücksichtigt werden. Dieser Beitrag thematisiert wichtige Aspekte des diagnostischen Vorgehens, der Prävention und der therapeutischen Möglichkeiten in Abhängigkeit von Ätiologie und Schwere des Handekzems.
\end{abstract}

$\begin{array}{ll}\begin{array}{l}\text { ABKÜRZUNGEN } \\ \text { BG-Verfahren } \\ \text { BZgA }\end{array} & \begin{array}{l}\text { Durchgangsarztverfahren } \\ \text { Bundeszentrale für gesundheit- } \\ \text { liche Aufklärung }\end{array} \\ \text { COVID-19 } & \begin{array}{l}\text { Coronavirus Disease 2019 } \\ \text { DKG }\end{array} \\ & \begin{array}{l}\text { Deutsche Kontaktallergie- } \\ \text { Gruppe }\end{array} \\ \text { FDA } & \text { Food and Drug Administration } \\ & \text { der USA } \\ \text { HECSI-75 } & \text { Hand Eczema Severity Index } \\ \text { IgE } & \text { Immunglobulin E } \\ \text { MCI } & \text { Chlor-Methylisothiazolinon } \\ \text { MI } & \text { Methylisothiazolinon } \\ \text { PUVA } & \text { Psoralen plus UV-A } \\ \text { RAR } & \text { Retinsäurerezeptor } \\ \text { RXR } & \text { Retinoid-X-Rezeptor } \\ \text { SIP } & \text { sekundäre Individualprävention } \\ \text { TIP } & \text { tertiäre Individualprävention } \\ \text { TIX } & \text { therapeutischer Index }\end{array}$

\section{Epidemiologie}

Handekzeme sind häufig und stellen den größten Anteil arbeitsbedingter Hauterkrankungen dar. Die Lebenszeitprävalenz beträgt ungefähr 10-15\% [1,2]. Epidemiologische Daten, die die Verbreitung des Handekzems in der Allgemeinbevölkerung beschreiben, stammen v.a. aus skandinavischen Ländern. Schwedische Daten lassen auf eine Inzidenz von 5-8\% (5,5-8,8 Fälle/1000 Personenjahre) schließen.
Interessanterweise war die Inzidenzrate bei Jugendlichen vergleichbar mit der von Erwachsenen, was den frühen Beginn der Erkrankung widerspiegelt. Die Inzidenzrate lag bei Frauen dabei etwas höher als bei Männern (7,1 vs. 4,0) [2]. Trotz der relativ geringen betroffenen Körperoberfläche haben Handekzeme eine hohe gesundheitsökonomische und sozialmedizinische Bedeutung sowie Auswirkungen auf alle Lebensbereiche. Patienten leiden häufig sowohl unter Schmerzen und Juckreiz wie auch unter den für ihre Umwelt sichtbaren, entstellenden Hautveränderungen der Hände.

\footnotetext{
Merke

Handekzeme haben negative psychosoziale Auswirkungen und führen zu einem Verlust an Lebensqualität.

\section{Risikofaktoren für Persistenz}

Die wichtigsten Risikofaktoren für die Persistenz von Handekzemen sind

- eine ausgeprägte Ausdehnung der Handekzeme,

- Handekzeme allergischer oder atopischer Genese,

- Ekzeme in der Kindheit und

- Beginn der Handekzeme vor dem 20. Lebensjahr [1].
} 
FALLBEISPIEL

\section{Fall 1}

Der 51 -jährige Herr B. berichtet über ein seit ca. 6 Jahren bestehendes Handekzem mit anamnestisch arbeitskongruentem Verlauf, eine deutliche Besserung sei zuletzt aber nur noch im Urlaub zu erzielen gewesen. Herr B. arbeitet seit über 30 Jahren als Werkzeugmacher, dabei hat er u. a. Kontakt zu Kühlschmierstoffen. Der Patient trägt ca. 3-4 Stunden pro Tag Nitrilhandschuhe, diese seien schnell durchfeuchtet, da er in ihnen schwitze. Dabei ist zu berücksichtigen, dass beim Um-



- Abb.1 Als Folge repetitiver Einwirkung von Irritanzien kam es zur Entstehung eines irritativen Handekzems, welches sich nach jahrelangem Bestehen morphologisch hyperkeratotisch-rhagadiform präsentiert (Quelle: Klinikum Nürnberg). gang mit rotierenden Maschinen arbeitsschutzrechtlich das Tragen von Handschuhen nicht zulässig ist. Eine atopische Diathese lässt sich nicht nachweisen.

Klinisch zeigt sich ein schweres Handekzem mit flächigen Hyperkeratosen und Rhagaden palmar und an den Handrücken beidseits.

\section{Procedere}

Ein BG-Verfahren wird mittels Hautarztbericht eingeleitet. In der Epikutantestung lassen sich keine berufsspezifischen Typ-IV-Sensibilisierungen, insbesondere gegenüber Kühlschmierstoffen, nachweisen. In Zusammenschau der Anamnese, des klinischen Bildes und der erhobenen Befunde kann ein irritatives Handekzem diagnostiziert werden, das morphologisch vorwiegend hyperkeratotisch-rhagadiform imponiert ( $\triangleright$ A b b. $\mathbf{1}$ ).

Es wird eine Lokaltherapie mit steroidhaltigen und salizylsäurehaltigen Externa eingeleitet, weiterhin erfolgt eine Therapie mit Creme-PUVA. Ein Therapieversuch mit Alitretinoin wird vom Patienten nicht gewünscht.

Am Arbeitsplatz wird der Patient zunehmend für Bürotätigkeiten eingesetzt. Darunter ergibt sich eine kontinuierliche Befundbesserung. Eine berufsdermatologische Betreuung besteht weiterhin.

\section{Ätiologie und Pathogenese}

Die Pathogenese des Handekzems ist multifaktoriell

Merke

Die drei häufigsten Faktoren, die zur Auslösung eines Handekzems führen, sind

- irritative Schädigung,

- Kontaktallergie und

- atopische Diathese.

Eine irritative Schädigung wird meist durch einen wiederholten Hautkontakt mit Wasser, eine mechanische Beanspruchung der Haut oder hautreizende Substanzen wie Detergenzien hervorgerufen. Kälte und Trockenheit, eine erhöhte Luftfeuchtigkeit bei Indoor-Arbeiten und der Kontakt zu Laugen und Säuren können ebenfalls das Auftreten eines Handekzems begünstigen.

Merke

Oft führt erst eine Kumulation mehrerer unterschwelliger Reize, v. a. im beruflichen Umfeld, zur Entstehung eines irritativen (subtoxisch-kumulativen) Handekzems.
Allen Handekzemformen gemeinsam ist, dass ihre Entstehung durch eine Störung der epidermalen Hautbarriere hervorgerufen wird. Durch einen vermehrten transepidermalen Wasserverlust kommt es zu einer Austrocknung der Haut. Dies erleichtert die Penetration von Infektionserregern, was zu Superinfektionen führen kann. Des Weiteren kommt es zu einer vermehrten Absorption von Kontaktallergenen, was die Entstehung einer allergischen Kontaktsensibilisierung begünstigt [3].

Dies bedingt die häufig beruflich assoziierten Pfropfsensibilisierungen, die auftreten können, wenn Patienten mit einem irritativen Handekzem in einem Arbeitsumfeld mit hoher Allergenexposition weiterarbeiten. Entsprechend der Pathogenese einer Kontaktallergie kommt es nach einer klinisch stummen, beschwerdefreien Sensibilisierungsphase bei erneuter Exposition mit dem Allergen zu einer massiven Inflammation. Spezifische T-Zellen werden in die Haut rekrutiert und aktivieren ihrerseits Leukozyten, eosinophile Granulozyten, Makrophagen etc. an den Ort des Geschehens. Klinisch präsentiert sich das Bild eines akuten Ekzems. 


\section{FAZIT}

\section{Take Home Message}

Arbeiten Patienten mit einem irritativen Handekzem in einem Arbeitsumfeld mit hoher Allergenexposition weiter, kann es zur Entstehung von Pfropfsensibilisierungen kommen.

In Zwillingsstudien konnte gezeigt werden, dass für das Risiko, Handekzeme zu entwickeln, zu $41 \%$ genetische Faktoren und zu 59\% Umweltfaktoren entscheidend sind. Eine besonders starke Assoziation konnte mit Loss-of-Function-Mutationen im Filaggrin-Gen nachgewiesen werden. Filaggrin ist entscheidend für die terminale Differenzierung der Korneozyten und trägt zur mechanischen Belastbarkeit der Hautbarriere bei. Mutationen des Filaggrin-Gens bei Atopikern sind mit einem frühen Beginn und einer Persistenz von Handekzemen, einem ungünstigen Krankheitsverlauf und häufiger Tätigkeitsaufgabe assoziiert [4].

Weitere Untersuchungen zeigen, dass es bei Patienten mit Handekzemen, unabhängig vom Subtyp des Handekzems, zu einer Herabregulierung zahlreicher Barriereproteine des sog. „cornified Envelope“ kommt. Als „cornified Envelope“ wird eine membranartige Struktur bezeichnet, welche die Korneozyten umgibt und aus zahlreichen Proteinen (u.a. Filaggrin, Loricrin) zusammengesetzt ist [5].

\section{Klinisches Bild}

Das Handekzem ist durch einen entzündlichen, nichtinfektiösen Hautausschlag an den Händen gekennzeichnet. Die Symptomatik und Morphologie sind dabei sehr variabel. Klinisch können sich u. a. Erytheme, Bläschenbildung, Hyperkeratosen, Rhagaden, entzündete Fingerkuppen und Lichenifikation zeigen. Viele Patienten berichten über brennende Schmerzen oder Pruritus.

Als akutes und subakutes Handekzem werden Ekzeme der Hände definiert, die weniger als 3 Monate andauern und nicht häufiger als 1-mal jährlich auftreten. Als chronisches Handekzem werden Handekzeme bezeichnet, die länger als 3 Monate andauern oder mindestens 2-mal pro Jahr rezidivieren $[2,6]$.

\section{FAZIT}

\section{Take Home Message}

Als chronisches Handekzem werden Handekzeme bezeichnet, die länger als 3 Monate andauern oder mindestens 2-mal pro Jahr rezidivieren.
\ Tab. 1 Einteilung der Handekzeme unter Berücksichtigung der Ätiologie.

\begin{tabular}{|l|l|}
\hline Exogene Handekzeme & Endogene Handekzeme \\
\hline $\begin{array}{l}\text { irritatives (subtoxisch-kumulatives) } \\
\text { Handekzem }\end{array}$ & atopisches Handekzem \\
\hline allergisches Handekzem & $\begin{array}{l}\text { hyperkeratotisch-rhagadiformes } \\
\text { Handekzem }\end{array}$ \\
\hline Protein-Kontaktdermatitis & $\begin{array}{l}\text { dyshidrotisches Handekzem/ } \\
\text { Pompholyx }\end{array}$ \\
\hline
\end{tabular}

Die Klassifikation des Handekzems beruht auf den unterschiedlichen Auslösemechanismen (exogene und endogene Auslöser) sowie auf den morphologischen Erscheinungsformen ( $\triangleright$ Tab. 1). Dabei ist zu berücksichtigen, dass häufig ein multifaktorielles Geschehen vorliegt und mehrere Ursachen gleichzeitig oder zeitlich versetzt von Bedeutung sein können. Dementsprechend ist die Systematik und Klassifikation des Handekzems eine Herausforderung, eine einheitliche evidenzbasierte Klassifikation existiert bisher nicht [6]. - Tab. 2 beschreibt die Charakteristika der drei häufigsten Handekzemtypen (irritatives, allergisches und atopisches Handekzem (s. a. > Abb. 2) [6, 7].

\section{FAZIT}

Take Home Message

Handekzeme sollten vorrangig anhand ihrer Ätiologie klassifiziert werden. Eine evidenzbasierte, allgemein akzeptierte Klassifikation existiert jedoch bisher nicht.

Es können jedoch auch Handekzeme mit dyshidrosiformer, hyperkeratotisch-rhagadiformer oder nummulärer Morphologie auftreten, die nicht eindeutig einer irritativen, allergischen oder atopischen Genese zugeordnet werden können. Diagnostische Schwierigkeiten können hier u.a. Handekzeme mit hyperkeratotischrhagadiformer Morphe bereiten, wenn ursächliche Faktoren nicht nachgewiesen werden können.

\section{PRAXIS}

Tipp

Es wird empfohlen, im Fall einer Dominanz der Morphologie in der Diagnosestellung zumindest einen Zusatz, z. B. hyperkeratotisch-rhagadiformes Handekzem genuiner Ätiologie oder unklarer Ätiologie hinzuzufügen [8]. Hyperkeratotischrhagadiforme Handekzeme sind dabei oft durch eine ausgeprägte Therapieresistenz und Rezidivneigung gekennzeichnet. 
- Tab.2 Charakteristika irritativer (subtoxisch-kumulativer), allergischer und atopischer Handekzeme [6]

\begin{tabular}{|c|c|c|c|}
\hline Subtyp & Ätiopathogenese & Lokalisation & Morphe \\
\hline $\begin{array}{l}\text { irritatives (subtoxisch- } \\
\text { kumulatives) Handekzem } \\
\text { ( }>\text { Abb.2a) }\end{array}$ & $\begin{array}{l}\text { - Folge wiederholter Einwirkung } \\
\text { von Irritanzien } \\
\text { - bei beruflicher Genese: kaum } \\
\text { Besserung am Wochenende, } \\
\text { Abheilung nur bei längerer } \\
\text { Arbeitskarenz } \\
\text { - begünstigende Faktoren: } \\
\text { atopische Diathese, Sebostase, } \\
\text { Hyperhidrose }\end{array}$ & $\begin{array}{l}\text { " vorwiegend Hand- und Finger- } \\
\text { rücken betroffen, ggf. exponierte } \\
\text { Unterarmpartien, erst im Verlauf } \\
\text { Handinnenflächen } \\
\text { - relativ scharfe Begrenzung }\end{array}$ & $\begin{array}{l}\text { - } \text { initial raue, trockene, schuppende } \\
\text { Haut } \\
\text { - später Rötung, Infiltration und } \\
\text { Rhagaden (hyperkeratotisch- } \\
\text { rhagadiformes Bild) }\end{array}$ \\
\hline $\begin{array}{l}\text { allergisches Handekzem } \\
\text { ( }>\text { Abb. } 2 \text { b) }\end{array}$ & $\begin{array}{l}\text { - Nachweis einer klinisch relevan- } \\
\text { ten Typ-IV-Sensibilisierung im } \\
\text { Epikutantest } \\
\text { - enger zeitlicher Zusammenhang } \\
\text { zwischen Erkrankung und Expo- } \\
\text { sition } \\
\text { - bei beruflichem Auslöser: Besse- } \\
\text { rung am Wochenende, Abheilen } \\
\text { im Urlaub, Rezidiv bei Rückkehr } \\
\text { an den Arbeitsplatz }\end{array}$ & $\begin{array}{l}\text { - betroffen sind die Expositions- } \\
\text { stellen } \\
\text { - unscharfe Begrenzung, Streureak- } \\
\text { tion in der Umgebung möglich }\end{array}$ & $\begin{array}{l}\text { - akutes Stadium: Rötung, Bläschen, } \\
\text { Juckreiz } \\
\text { - chronisches Stadium: Hyperkera- } \\
\text { tosen, Rhagaden }\end{array}$ \\
\hline $\begin{array}{l}\text { atopisches Handekzem } \\
(>\text { Abb. } \mathbf{2 c}, \nabla \text { Abb. } 2 \text { d) }\end{array}$ & $\begin{array}{l}\text { - bekanntes atopisches Ekzem } \\
\text { oder atopische Hautdiathese } \\
\text { - häufig berufsunabhängiger Ver- } \\
\text { lauf, aber Verschlechterung } \\
\text { durch berufliche Trigger möglich }\end{array}$ & $\begin{array}{l}\text { - häufig Manifestation am Hand- } \\
\text { rücken } \\
\text { - ggf. Nagelbeteiligung und Finger- } \\
\text { kuppenekzeme } \\
\text { - Ekzeme auch an anderen Prädilek- } \\
\text { tionsstellen }\end{array}$ & $\begin{array}{l}\text { - häufig dyshidrosiforme Bläschen } \\
\text { palmar und interdigital mit Aus- } \\
\text { breitung von der distalen Palmar- } \\
\text { region auf die proximalen Finger- } \\
\text { glieder zweier benachbarter } \\
\text { Finger (sog. „Apron Pattern“) } \\
\text { - Lichenifikation, Schuppung, } \\
\text { Rhagaden } \\
\text { - nummuläre Herde möglich }\end{array}$ \\
\hline
\end{tabular}

Beim dyshidrotischen Ekzem (Maximalvariante: Pompholyx) handelt es sich um ein Handekzem mit rezidivierend auftretenden, vesikulären Eruptionen und Erythemen insbesondere an den Fingerkanten, welches mit ausgeprägtem Pruritus einhergeht. Die Ätiologie ist nicht gut verstanden. Als auslösende Faktoren werden eine Atopie sowie eine Tinea corporis diskutiert, auch kontaktallergische Reaktionen können ein relevanter ätiologischer Faktor sein. [9].

Eine weitere Sonderform stellt die Proteinkontaktdermatitis dar. Sie ist Folge einer Typ-I-Sensibilisierung gegenüber u. a. Nahrungsmittelallergenen und findet sich bei entsprechender beruflicher Exposition [10]. Es handelt sich um die Kombination einer Sofort- mit einer Spättypreaktionen, die sich klinisch initial häufig als lokalisierte Urtikaria manifestiert, im Verlauf dominiert eine Ekzemmorphe. Hautpricktestungen mit dem vermuteten Auslöser, die Bestimmung spezifischer IgE-Antikörper und Epikutan- bzw. Expositionstestungen werden diagnostisch eingesetzt.

\section{Diagnostik}

\section{Anamnese}

Zur Diagnostik von Handekzemen ist eine detaillierte Anamneseerhebung erforderlich. Diese sollte insbesondere folgende Punkte umfassen:

- bekannte Allergien und Hauterkrankungen, insbesondere atopische Erkrankungen,

- spezifische Exposition im beruflichen und privaten Umfeld, Dauer und Zeitverlauf des Handekzems (insbesondere unter Beachtung arbeitsfreier Zeiten),

- Medikamenteneinnahme und Grunderkrankungen,

- Nikotinkonsum,

- Feuchtarbeit als Risikofaktor für die Entstehung von Handekzemen; diese kann anamnestisch anhand folgender Kriterien konstatiert werden:

- direkter Hautkontakt mit Wasser (>2 h/Tag),

- Tragen feuchtigkeitsdichter Handschuhe (>2 h/ Tag),

- Frequenz und Intensität der Händewaschfrequenz (>10×/Tag),

- ggf. spezielle berufliche Anamnese bezüglich Hautschutzmittel und Schutzhandschuhe. 


\section{Hautinspektion}

Neben der Inspektion der Hände sollte zur Abgrenzung von Differenzialdiagnosen (s.u.: Übersicht „Wichtige Differenzialdiagnosen des Handekzems") zusätzlich eine Ganzkörperinspektion der Haut (einschließlich Füße) erfolgen.

\section{Atopie-Screening}

Zur Erfassung einer atopischen Hautdiathese ist die Erhebung des Erlanger Atopie-Scores hilfreich [11]. Eine Pricktestung oder eine Bestimmung spezifischer IgE-Antikörper auf häufige Inhalationsallergene können ergänzend erfolgen, um ggf. eine atopische Diathese zu untermauern.

\section{Epikutantestung}

\section{Merke}

Bei allen länger als 3 Monaten bestehenden Handekzemen und/oder rezidivierendem Verlauf ist eine allergologische Abklärung mittels Epikutantestung erforderlich.

Bezüglich der Durchführung und Auswertung des Epikutantests mit Kontaktallergenen wird auf die aktuelle S3-Leitlinie verwiesen [12]. Die Testung der Standardreihe, in welcher die häufigsten Kontaktallergene zusammengefasst sind, sollte zusätzlich zu einer anamnesegeleiteten Auswahl weiterer Testallergene erfolgen. Bei Verdacht auf eine arbeitsbedingte Typ-IV-Allergie existieren für einzelne, besonders gefährdete Berufsgruppen expositionsbezogene Testungsempfehlungen $[13,14]$. Darüber hinaus kann die Testung patienteneigener Substanzen notwendig sein.

In einer multizentrischen europäischen Untersuchung waren mit Reaktionen von jeweils über $10 \%$ Nickel, (Chlor-)Methylisothiazolinon (MCl/MI), Duftstoffmix I, Kobalt, Thiuram-Mix, Perubalsam, Chrom und Duftstoffmix II die am häufigsten erkannten Kontaktallergene bei Handekzempatienten [15].

Für jede nachgewiesene Kontaktsensibilisierung muss eine individuelle Relevanzbeurteilung erfolgen. Berufliche und private Expositionsquellen sollten identifiziert und der Patient über erforderliche Karenzmaßnahmen aufgeklärt werden.

\section{FAZIT}

\section{Take Home Message}

Eine individuelle Relevanzbeurteilung ist für jede nachgewiesene Kontaktsensibilisierung erforderlich.

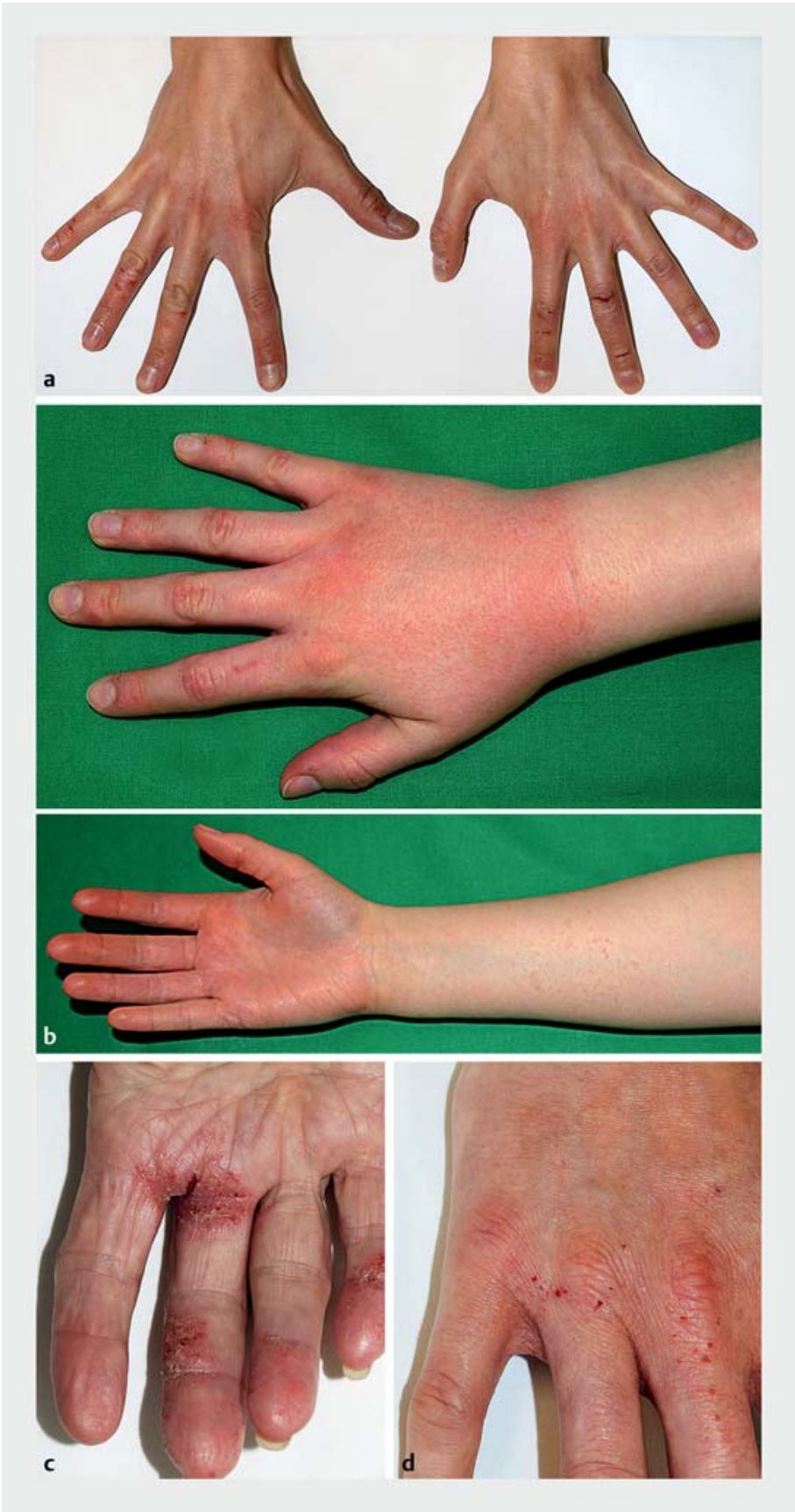

- Abb. 2 Klinisches Bild des irritativen (subtoxisch-kumulativen), allergischen und atopischen Handekzems (Quelle: Klinikum Nürnberg). a Schweres, irritatives (kumulativ-subtoxisches) Handekzem bei einer Reinigungskraft, es sind überwiegend die Handrücken betroffen. b Allergisches Handekzem, ausgelöst durch das Tragen von Gummihandschuhen bei Typ-IV-Allergie gegenüber Thiuramen (Gummiinhaltsstoffen). Typische Streureaktionen sind am Unterarm volar sichtbar. c Atopisches Handekzem, das sich überwiegend mit nummulären Ekzemherden präsentiert. d Vorwiegend dyshidrosiforme Bläschen bei einem Patienten mit atopischem Handekzem. 


\section{FALLBEISPIEL}

\section{Fall 2}

Der 69-jährige Herr L. berichtet, seit 7 Monaten unter stark juckenden „Wasserbläschen“, v. a. an den Händen, in geringerem Umfang auch an den Füßen, zu leiden. Bei Inspektion zeigen sich ausgedehnte Bläschen, Blasen, Erosionen und Krusten auf erythematösem Grund sowohl palmar als auch an den Handrücken (in $>$ Abb. $\mathbf{3}$ sind nach Abheilung der Bläschen lediglich noch Erosionen und Krusten sichtbar). Der Erlanger Atopiescore lässt auf eine atopische Diathese schließen. Der Patient ist Rentner, Hinweise auf eine irritative Genese ergeben sich nicht. Im Epikutantest können relevante Kontaktsensibilisierungen ausgeschlossen werden.

\section{Diagnose}

In Zusammenschau des klinischen Bildes, der Anamnese und der erhobenen Befunde ist von einem atopischen Handekzem auszugehen.

\section{Therapie und Verlauf}

Therapeutisch erfolgt zunächst eine stadiengerechte, steroidhaltige und adstringierende Lokaltherapie in Kombination mit einer Creme-PUVA-Lichttherapie. Bei unzureichender Befundbesserung wird eine orale Therapie mit Ciclosporin eingeleitet, diese muss jedoch nach 4 Wochen bei entgleister arterieller Hypertonie wieder abgesetzt werden. Daraufhin wird der Patient unter regelmäßiger laborchemischer Kontrolle der Leberparameter, Schilddrüsenwerte und Blutfettwerte auf Alitretinoin in einer Dosierung von $30 \mathrm{mg}$ eingestellt. Bereits nach 3 Wochen ist eine wesentliche Befundbesserung zu erkennen, nach ca. 6 Monaten ist das Handekzem nahezu abgeheilt ( $>$ Abb.4), sodass die Therapie beendet werden kann.
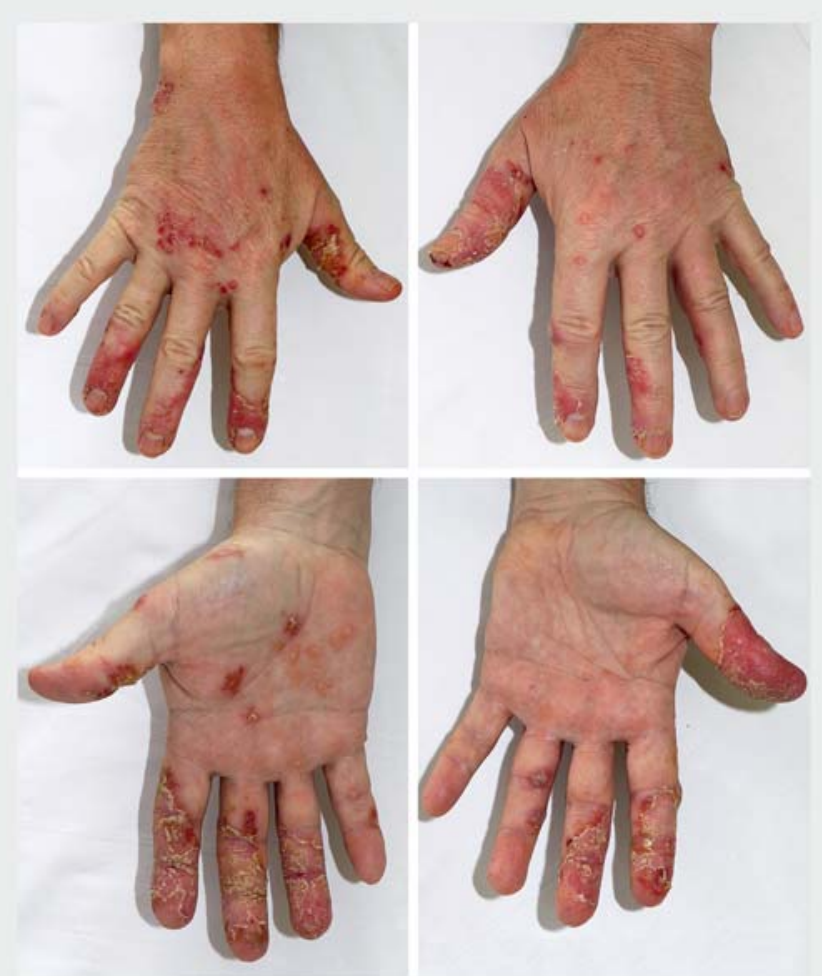

Abb. 3 Zustand vor Behandlungsbeginn mit Alitretinoin (Quelle: Klinikum Nürnberg).
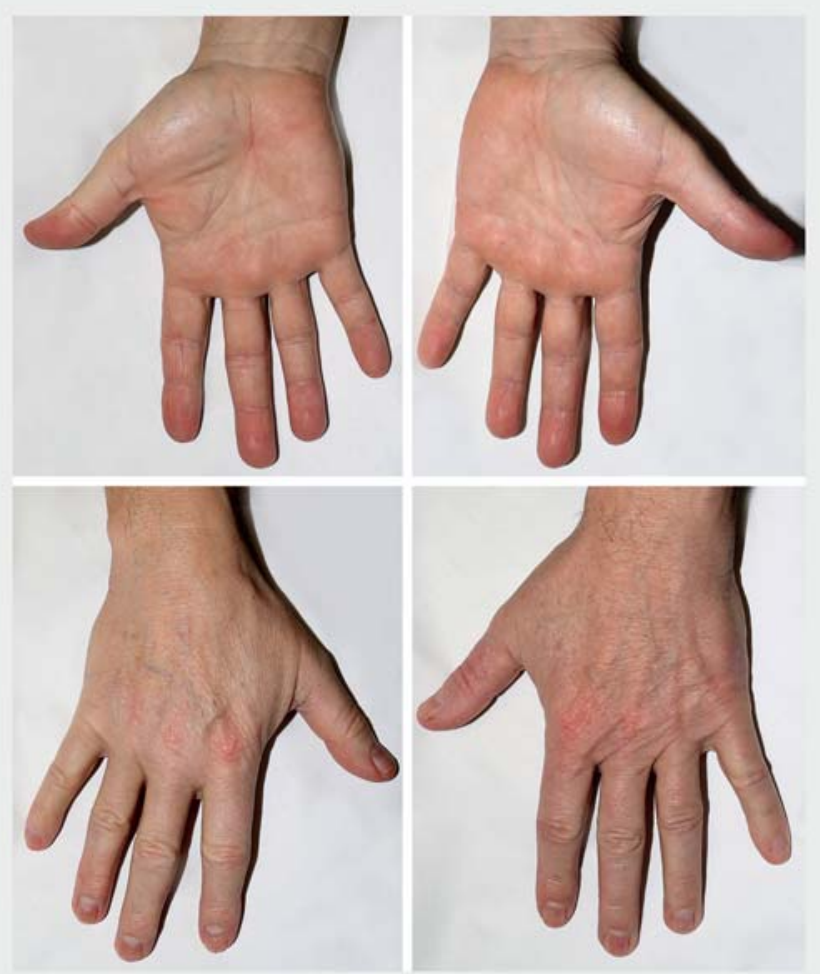

Abb. 4 Nahezu abgeheilten Hautbefund ca. 6 Monate nach der Therapie mit Alitretinoin (Quelle: Klinikum Nürnberg). 


\section{Weitere Diagnostik}

Eine Auswahl wichtiger Differenzialdiagnosen des Handekzems ist in der u.g. Übersicht zusammengefasst.

Zum Ausschluss einer Tinea manuum sollte initial eine mykologische Diagnostik erfolgen. Bei Hinweis auf das Vorliegen einer Differenzialdiagnose ist die Entnahme einer Probebiopsie zur histologischen Untersuchung zu empfehlen.

Dabei ist die histologische Differenzialdiagnose Psoriasis palmoplantaris vs. chronisches Handekzem oft schwierig. Kürzlich konnten jedoch genetische Biomarker wie NOS2 (hochreguliert in Psoriasisherden, kodiert für die induzierbare NO-Synthase) und CCL27 (kutanes T-Zell-Chemokin, hochreguliert bei Patienten mit Ekzemen) identifiziert werden, welche mithilfe eines molekularen Tests in befallener Haut mit einer hohen Sensitivität und Spezifität beide Krankheitsbilder unterscheiden können. Diese sind für die Routinediagnostik noch nicht verfügbar, können jedoch zukünftig das diagnostische Spektrum erweitern und ggf. personalisierte Therapieentscheidungen unterstützen [16, 17].

Wichtige Differenzialdiagnosen des Handekzems fasst die Übersicht zusammen.



\section{Therapie}

Der Einsatz einer Lokaltherapie, physikalischen Therapie und Systemtherapie sollte in Abhängigkeit von der Ätiologie und vom Schweregrad des Handekzems erfolgen. Unterstützend sind Patientenschulungen sinnvoll, da die Vermeidung des Kontaktes zu Allergenen und Noxen sowie die Umsetzung von Hautschutzmaßnahmen entscheidend zum Therapieerfolg beitragen. Eine Übersicht zur stadienadaptierten Stufentherapie, entsprechend dem Schweregrad des Handekzems, ist in Tab.3 dargestellt. Insbesondere bei schweren, chronischen Handekzemen ist oft ein multimodales Therapiekonzept mit einer Kombination aus Basistherapie, Lokaltherapie, UV-Phototherapie und Systemtherapie erforderlich $[6,7,18]$.

FAZIT

Take Home Message

Die Therapie des Handekzems erfolgt stadienadaptiert, häufig ist ein multimodales Therapiekonzept erforderlich ( $\triangleright$ Tab.3).

\section{Lokaltherapien}

Basistherapie

Hautpflegeprodukte sollte idealerweise täglich bei allen Patienten mit chronischem Handekzem angewendet werden. Die Therapie der Xerosis cutis soll die Hydratation der Haut verbessern und damit die Barrierefunktion der Haut unterstützen. Es stehen verschiedene Produkte zur Rückfettung mit z.B. Urea, Milchsäurederivaten und Glycerin zur Verfügung. Bei der Auswahl sollten die Grundlagen der topischen Therapie, der individuelle Hautzustand und bekannte Typ-IV-Sensibilisierungen z.B. auf Konservierungsstoffe berücksichtigt werden [19].

Als keratolytische Lokaltherapie bei überwiegend hyperkeratotischen Handekzemen können ureahaltige Lokaltherapeutika in einer Konzentration von 10-20\% eingesetzt werden. Der Effekt kann durch eine okklusive Behandlung gesteigert werden. Weiters können salizylsäurehaltige Externa in einer Dosierung von 3-20\% in unterschiedlichen Grundlagen zur Keratolyse verwendet werden. Bei der Verwendung von Magistralrezepturen sollte die „Empfehlung zur Qualitätssicherung: Wirkstoffdossiers für externe dermatologische Rezepturen der Gesellschaft für Dermopharmazie“ berücksichtigt werden. Hier sind u. a. valide Informationen zur therapeutischen Breite von Wirkstoffen aufgeführt [20].

\section{Glukokortikosteroide}

\footnotetext{
Merke

Topische Glukokortikosteroide stellen gemeinsam mit der Basistherapie i.d. R. die erste Therapieoption beim Handekzem dar, ihre Wirksamkeit ist evidenzbasiert [21].
}

Sie sind in der Akuttherapie sehr effektiv, verzögern aber die Reparaturmechanismen des Stratum corneum und können bei unsachgemäßer Anwendung potenziell Hautatrophien verursachen [6]. 
- Tab.3 Stadienadaptierte Therapie des Handekzems $[6,7,18]$.

\begin{tabular}{|c|c|c|}
\hline Therapiestufe & Schweregrad des Handekzems & Therapeutische Maßnahmen \\
\hline Basistherapie & Xerosis cutis & $\begin{array}{l}\text { Hydratation der Haut (s. Infobox „Hautmittel und Hautschutzmaßnahmen“) } \\
\text { Einsatz von Hautschutzmaßnahmen (s. Infobox „Hautmittel und Hautschutz- } \\
\text { maßnahmen“) } \\
\text { Reduktion/Vermeidung von Triggerfaktoren }\end{array}$ \\
\hline I & $\begin{array}{l}\text { leichte Handekzeme } \\
\text { (heilen bei adäquater dermato- } \\
\text { logischer Therapie und Mitwir- } \\
\text { kung des Patienten rasch wieder } \\
\text { ab) }\end{array}$ & $\begin{array}{l}\text { zusätzlich zur Basistherapie: } \\
\text { " topische Glukokortikosteroide } \\
\text { " topische Calcineurin-Inhibitoren (zugelassen nur beim atopischen } \\
\text { Handekzem) } \\
\text { - antipruriginöse und antiseptische Externa } \\
\text { - Leitungswasser-Iontophorese }\end{array}$ \\
\hline II & $\begin{array}{l}\text { mittelschwere und schwere } \\
\text { Handekzeme } \\
\text { (bestehen trotz adäquater } \\
\text { dermatologischer Therapie } \\
\text { und Mitwirkung des Patienten } \\
\text { mehrere Wochen) }\end{array}$ & $\begin{array}{l}\text { zusätzlich zu Maßnahmen der Stufe I: } \\
\text { " } \text { topische potente Glukokortikosteroide } \\
\text { " UV-Therapie (Creme- oder Bade-PUVA, UVB) } \\
\text { - } \text { systemisch Alitretinoin (zugelassen nur bei schweren Handekzemen) }\end{array}$ \\
\hline III & $\begin{array}{l}\text { chronisch-rezidivierende und } \\
\text { persistierende Handekzeme } \\
\text { (ausgedehnte dauerhaft vor- } \\
\text { handene oder rezidivierende } \\
\text { Hautveränderung von erheb- } \\
\text { lichem Krankheitswert mit z. B. } \\
\text { Rhagaden, ausgeprägter Liche- } \\
\text { nifikation und Infiltration) }\end{array}$ & $\begin{array}{l}\text { zusätzlich zu Maßnahmen der Stufe I und II: } \\
\text { - Alitretinoin } \\
\text { - Ciclosporin (zugelassen nur beim atopischen Handekzem) } \\
\text { - systemische Glukokortikosteroide (nur kurzfristig im akuten Schub) } \\
\text { - ggf. weitere Systemtherapeutika } \\
\text { - Dupilumab (zugelassen beim atopischen Ekzem) }\end{array}$ \\
\hline
\end{tabular}

Die Präparate mit einem guten therapeutischen Index (TIX) sind zu bevorzugen, da sie ein gutes Verhältnis von erwünschten zu unerwünschten Wirkungen erzielen [22]. Dazu gehören z. B.

- Prednicarbat (Klasse II, TIX 2,0),

- Methylprednisolonaceponat (Klasse III, TIX 2,0) oder

- Mometasonfuroat (Klasse III, TIX 2,0).

Ein Rebound-Phänomen sollte durch gezieltes Ausschleichen der Anwendungsfrequenz vermieden werden.

Insgesamt sollten potente Glukokortikosteroide nicht länger als $1 \times$ täglich über 4 Wochen angewendet werden. Anschließend sollte eine Reduktion der Anwendungsfrequenz erfolgen bis zu einer Erhaltungstherapie mit 2-3×wöchentlicher Applikation (sogenannte proaktive Therapie, Anwendung z. B. für 6 Wochen) [23].

\section{Topische Calcineurin-Inhibitoren}

Der Einsatz der topischen Calcineurin-Inhibitoren Tacrolimus und Pimecrolimus ist zur Behandlung des mittelschweren bis schweren atopischen Ekzems bei Erwachsenen indiziert. Sie können auch zur Therapie des Handekzems eingesetzt werden, wobei sie hierfür nicht zugelassen sind (Off-Label-Use) $[6,18]$. Da Tacrolimus nur in einer Salbengrundlage und Pimecrolimus ausschließlich als Creme verfügbar ist, sollte die Galenik berücksichtigt werden. Die Behandlung erfolgt initial $2 \times$ täglich, bei Abheilung Reduktion auf $1 \times$ tägliche An- wendung, gefolgt von einer Erhaltungstherapie jeden zweiten Tag für weitere 6 Wochen (proaktive Therapie).

Entsprechend der „Black Box“-Warnung der FDA sollte eine Kombination mit künstlichem und natürlichem UV-Licht vermieden werden [24]. Die begleitende Anwendung von topischem und textilem Lichtschutz ist sinnvoll, auch wenn es keine sichere Evidenz gibt, die den Zusammenhang zwischen Hautkrebs bzw. Lymphomen und der Anwendung topischer Calcineurin-Inhibitoren belegt [18].

\section{Sonstige Lokaltherapeutika}

Bei Superinfektion sind antiseptische und desinfizierende Wirkstoffe wie Chlorhexidin, Clioquinol und Octenidin, z. B. als Waschlotion oder Handbad, hilfreich. Bei nässenden Ekzemen können Gerbstoffe lokaltherapeutisch eingesetzt werden [25]. Sie sind in verschiedenen Grundlagen verfügbar, z.B. Badezusatz, Creme oder Fettcreme.

Steinkohleteerpräparate sind in der Vergangenheit häufig zur Behandlung des atopischen Ekzems und Handekzems eingesetzt worden. Positive Effekte auf die Filaggrin-Expression wurden beschrieben $[23,26]$. Aufgrund der potenziellen Kanzerogenität und der kosmetischen Einschränkungen wurde Steinkohleteer zuletzt jedoch kaum noch in der Behandlung des Handekzems eingesetzt $[18,23]$. 


\section{Systemtherapien}

\section{Alitretinoin}

Alitretinoin (9-cis-Retinsäure) ist seit 2008 zugelassen zur Therapie des schweren chronischen Handekzems bei Erwachsenen, die nicht auf eine Behandlung mit potenten topischen Kortikosteroiden ansprechen. Es wirkt als Agonist sowohl auf den Retinsäurerezeptor (RAR) als auch auf den Retinoid-X-Rezeptor (RXR). Die Wirkung von Alitretinoin ist antiinflammatorisch und immunmodulierend. In den klinischen Zulassungsstudien zeigten sich positive Effekte auf alle Handekzemtypen, wobei Patienten mit hyperkeratotischen Handekzemen am meisten von der Therapie profitierten [27].

Die Standarddosierung beträgt $30 \mathrm{mg} /$ Tag p.o. und kann bei persistierenden und nicht ausreichend kontrollierten Nebenwirkungen auf $10 \mathrm{mg} /$ Tag reduziert werden. Ein Behandlungszyklus dauert i.d.R. 12-24 Wochen. Bei Rezidiv können Patienten von einem weiteren Behandlungszyklus profitieren.

Alitretinoin ist als Vitamin-A-Säure-Derivat teratogen und deswegen prinzipiell bei Frauen im gebärfähigen Alter kontraindiziert. Eingesetzt werden kann es in Einzelfällen dennoch, wenn einen Monat vor Therapiebeginn, während der Therapie und bis einen Monat nach Absetzen der Therapie ein striktes Schwangerschaftspräventionsprogramm mit sicherer Kontrazeption befolgt wird. In der Stillzeit darf es nicht eingesetzt werden. Als Kontraindikation gilt darüber hinaus eine bekannte Soja- und Erdnussallergie. Alitretinoin sollte nicht zusammen mit Methotrexat oder Tetrazyklinen eingenommen werden.

Cave

Alitretinoin ist teratogen, sodass bei Patientinnen im gebärfähigen Alter eine sichere Kontrazeption zu gewährleisten ist.

Die häufigste Nebenwirkung sind Kopfschmerzen (bei ca. $20 \%$ der Patienten) insbesondere in den ersten beiden Therapiewochen. Die Symptomatik ist dosisabhängig und i.d.R. vorübergehend. Regelmäßige laborchemische Kontrollen insbesondere der Blutfettwerte (Triglyzeride, Cholesterin), Schilddrüsenparameter und Transaminasen sind erforderlich. Bezüglich Gegenanzeigen und besonderer Warnhinweise wird auf die Fachinformation verwiesen.

\section{Ciclosporin}

Ciclosporin ist zur Therapie des schweren, therapieresistenten, atopischen Ekzems bei Erwachsenen seit 1997 zugelassen. Zur Therapie des Handekzems ist es nicht zugelassen. Patienten mit atopischem Handekzem sprechen i.d.R. gut auf eine Ciclosporin-Therapie an [21]. Aufgrund von Nebenwirkungen (u.a. arte- rielle Hypertonie, Nierenfunktionsstörungen) wird Ciclosporin im klinischen Alltag jedoch eher zurückhaltend eingesetzt. Die Verabreichung erfolgt in einer Dosierung von $2,5-5 \mathrm{mg} / \mathrm{kgKG} / \mathrm{Tag}$ verteilt auf 2 Einzeldosen. Die Kombination mit einer Lichttherapie ist kontraindiziert.

\section{Systemische Glukokortikosteroide}

Systemische Glukokortikosteroide (0,5-1 mg/kgKG) Tag Prednisolonäquivalent) können kurzfristig zur Behandlung ausgeprägter, akuter Handekzemschübe eingesetzt werden. Sie sind aber zur längerfristigen Behandlung chronischer Handekzeme aufgrund ihres Nebenwirkungsprofils ungeeignet [6].

\section{Neue Systemtherapien}

Dupilumab, ein II-4/13-Blocker, ist seit 2017 für die Therapie des mittelschweren bis schweren atopischen Ekzems zugelassen, jedoch nicht für die Therapie eines isoliert auftretenden atopischen Handekzems. Oesterhaven et al. untersuchten das therapeutische Ansprechen bei einer Kohorte von 47 Patienten mit atopischem Ekzem, die ebenfalls ein atopisches Handekzem aufwiesen. Bei $60 \%$ der untersuchten Patienten konnte eine mindestens $75 \%$ ige Verbesserung des HECSI-75 (Hand Eczema Severity Index) nach 16 Wochen erreicht werden [28]. Dementsprechend scheint Dupilumab eine Therapieoption für Patienten mit zusätzlich bestehendem atopischem Ekzem zu sein. Weitere Studien sind jedoch erforderlich, um die Effektivität auch bei Patienten mit anderen Handekzemformen zu untersuchen.

Der Januskinase-Inhibitor Baricitinib ist seit Oktober 2020 für die Behandlung Erwachsener mit mittelschwerem bis schwerem atopischem Ekzem zugelassen [29]. Weitere Studien müssen zeigen, ob ein therapeutischer Einsatz auch beim chronischen Handekzem effektiv ist. Erste Berichte gibt es über den topischen Einsatz des pan-JAK-Inhibitors Delgocitinib [30].

\section{Weitere systemische Therapien}

Weitere in Einzelfällen eingesetzte Therapeutika ohne Zulassung (Off-Label-Use) sind Azathioprin und Methotrexat. Die Verwendung von Acitretin kann bei hyperkeratotischen Hand- und Fußekzemen erwogen werden. Als Vitamin-A-Säure-Derivat ist Acitretin teratogen und streng kontraindiziert bei Schwangeren und Frauen im gebärfähigen Alter (Schwangerschaftsverhütung bis einschließlich 3 Jahre nach beendeter Behandlung erforderlich). Auf die entsprechende Fachinformation wird verwiesen. 


\section{FALLBEISPIEL}

\section{Fall 3}

Der 33-jährige Herr D. arbeitet seit 11 Jahren als Industriemechaniker. Seit einem halben Jahr treten Juckreiz, Bläschen und Rötungen an den Händen und Unterarmen nach Kontakt zu einem bestimmten Konstruktionsklebstoff auf. Zudem leidet er zuletzt zeitgleich auch unter Rötungen und Schwellungen im Gesichtsbereich (vgl. \ Abb.5). Der Patient trägt keine Handschuhe, die Schutzbrille ist seitlich offen. Bei beruflichen Tätigkeiten ohne Kontakt zu Klebern besteht Symptomfreiheit.

\section{Procedere}

Ein Hautarztverfahren wird aufgrund der Arbeitskongruenz mittels Hautarztbericht eingeleitet. Es erfolgt eine Epikutan-


- Abb.5 Allergisches Handekzem und aerogenes allergisches Kontaktekzem mit Erythemen und z. T. Vesiculae (Quelle: Klinikum Nürnberg). a Klinisches Bild am Unterarm des Patienten (ähnliches Bild auch an den Händen). b Gesichtsbereich. testung mit den DKG-Reihen Standard, Kunstharze/Kleber und Bau-Hauptgewerbe. Hier lassen sich Typ-IV-Sensibilisierungen auf folgende Komponenten in Epoxidharzsystemen nachweisen:

- 1,4-Butandiol-diglycidylether (Reaktivverdünner),

- 1,6-Hexandiol-diglycidylether (Reaktivverdünner) und

- Trimethylhexan-1,6-diamin (Härter).

Dementsprechend kann ein aerogenes allergisches Kontaktekzem und allergisches Handekzem bei Typ-IV-Allergie auf Härter und Reaktivverdünner von Epoxidharzen diagnostiziert werden. Epoxidharze sind potente Kontaktallergene und weisen eine hohe Sensibilisierungspotenz auf. Sie werden u. a. in der Kunststoffindustrie, als Bindemittel von Klebstoffen, in Farben und Schutzanstrichen verwendet. Herauszustellen ist, dass bei ausschließlicher Testung der Standardreihe inklusive dem darin enthaltenen Epoxidharz die relevanten Kontaktallergene nicht identifiziert worden wären. Es sollten bei berufsassoziierten Handekzemen demzufolge immer berufsspezifische Reihen mitgetestet werden.

Bei Verdacht auf eine Epoxidharzallergie sollten Härter und Reaktivverdünner (DKG-Reihe Kunstharze/Kleber) mitgetestet werden.

Bei Herrn D. sistieren die Symptome nach Meidung des Kontaktes zu Konstruktionsklebstoffen vollständig.

\section{Physikalische Therapie}

\section{UV-Lichttherapie}

Bei chronisch-rezidivierenden Verlauf des Handekzems ist bei Erwachsenen ab Therapiestufe II eine Lichttherapie indiziert (UVB, Creme- oder Bade-PUVA). PUVATherapie ist dabei gemäß einem aktuellen Cochrane Review einer Schmalspektrum-UVB-Lichttherapie überlegen [18,21]. Aufgrund der guten Praktikabilität kommt meist die Creme-PUVA-Therapie zur Anwendung. Eine Kombination mit Retinoiden (einschließlich Alitretinoin) kann synergistisch wirken.

\section{Leitungswasser-Iontophorese}

Bei der Leitungswasser-Iontophorese handelt es sich um eine galvanische Methode zur Behandlung der palmaren und plantaren Hyperhidrose sowie des dyshidrosiformen Handekzems. Nach einer initial täglichen Anwendung kann die Frequenz im Verlauf langsam reduziert werden, allerdings sind Rezidive nach Beendigung der Therapie häufig.

\section{Prävention/berufliche Aspekte}

Allen Patienten mit Handekzemen wird eine Veränderung des Lebensstils und der Gewohnheiten empfohlen. Identifizierte Allergene und Irritanzien sollen vermieden und möglichst durch andere Stoffe ersetzt werden, des Weiteren sollen Hautschutzmaßnahmen konsequent angewendet und Feuchtarbeiten sowie mechanische Irritationen vermieden werden ([19,31,32]; s. a. Infobox).

Beruflich verursachte oder verschlimmerte Handekzeme können zu einer Berufskrankheit (BK-Nr. 5101) führen. Dabei repräsentieren sie den überwiegenden Anteil an Fällen dieser Berufskrankheit und machen etwa ein Drittel aller angezeigten Berufskrankheiten aus. 
Insbesondere bei Hochrisikoberufen (u.a. Gesundheits- und Pflegeberufe, Metallarbeiter und Mechaniker, Küchenberufe, Baugewerbe, Druckereitätigkeiten, Friseure und Kosmetiker) geht der Kontakt zu hautgefährdenden Stoffen (welche bspw. ätzende, irritative und sensibilisierende Eigenschaft haben können) sowie die Feuchtarbeit mit einem erhöhten Handekzemrisiko einher [33].

Zur Prävention der Entstehung einer Berufskrankheit wurde das „Hautarztverfahren“ geschaffen, welches mittels Hautarztbericht eingeleitet werden kann. Der zuständige Unfallversicherungsträger soll hiermit frühzeitig informiert werden, sodass entsprechende Präventionsmaßnahmen getroffen werden können. Diese umfassen neben der Anwendung von Hautschutz-, Hautpflege- und Hautreinigungsmitteln auch geeignete Schutzhandschuhe (s. Infobox). Bei der Versorgung mit geeigneten Handschuhen müssen die individuellen Arbeitsstoffe und Arbeitsanforderungen berücksichtigt werden.

Zum 1. Januar 2021 ist eine Änderung des Berufskrankheitenrechts in Kraft getreten, die auch für dermatologische Erkrankungen eine große Bedeutung hat. Während zuvor die Aufgabe der schädigenden Tätigkeit („Unterlassungszwang“) eine Voraussetzung dafür war, dass die Berufskrankheit anerkannt wurde, so fällt diese Hürde nun weg. Maßgeblich sind jetzt ausschließlich die Schwere (z. B. Persistenz eines Handekzems trotz stadiengerechter Therapie über 6 Monate) oder die wiederholte Rückfälligkeit der Erkrankung. Damit kann nun sehr viel früher als bisher eine Berufskrankheitenanzeige gestellt werden [34].

\section{FAZIT}

\section{Take Home Message}

Das Hautarztverfahren soll die Entstehung einer Berufskrankheit BK 5101 verhindern und wird meist mittels Hautarztbericht eingeleitet. Besteht der begründete Verdacht, dass eine BK 5101 vorliegt sollte eine Berufskrankheitenanzeige erfolgen.

Durch zusätzliche Angebote der Unfallversicherungsträger im Rahmen einer sekundären Individualprävention (SIP; ambulante Hautschutzschulung) und der tertiären Individualprävention (TIP; stationärer Aufenthalt in einer berufsdermatologischen Schwerpunktklinik mit anschließender nachstationärer Phase der Arbeitskarenz unter ambulanter hautfachärztlicher Betreuung) kann bei der Mehrheit der Patienten eine Besserung des Hautbefundes und damit ein Berufsverbleib erzielt werden [35].

\section{INFOBOX}

\section{Hautmittel und Hautschutzmaßnahmen}

Zum Hautschutzkonzept am Arbeitsplatz gehören neben Schutzhandschuhen die beruflichen Hautmittel. In Deutschland werden dazu Hautschutz, Hautpflege und Hautreinigungspräparate gezählt, die i.d.R. in Kombination eingesetzt werden [29].

\section{Generelle Hautschutzmaßnahmen}

- Bei Feuchtarbeiten sollten Schutzhandschuhe (so oft wie nötig und so kurz wie möglich) getragen werden.

- Schutzhandschuhe sollten sauber, intakt und auf der Innenseite trocken sein.

- Bei Tragedauer > 10 Minuten sollten Baumwollhandschuhe unter den Schutzhandschuhen getragen werden.

- Fingerringe sollten während der Arbeit nicht getragen werden

- Zum Geschirrspülen sollten Schutzhandschuhe über Baumwollhandschuhen getragen werden.

\section{Hautschutzpräparate}

- Anwendung während der Arbeitszeit

- Verringerung von Hautirritationen durch Arbeitsstoffe

- Stabilisierung der Hautbarriere bei Feuchtbelastungen

- erleichtertes Entfernen von Verschmutzungen

\section{Hautreinigung und Händedesinfektion}

- Eine hohe Handwaschfrequenz stellt einen Risikofaktor für die Entstehung von Handekzemen dar.

- Der Einsatz milder Reinigungsprodukte mit optimalem $\mathrm{pH}$ wird empfohlen (pH 5,5).

- Bevorzugung von alkoholischer Händedesinfektion (mit rückfettendem Zusatz), da dies die Haut weniger irritiert als eine Reinigung mit Seife.

\section{Hautpflege}

- Regeneration der Hautbarriere

- Einsatz bevorzugt nach der Arbeit

- Verteilung auf der gesamten Handoberfläche inkl. Interdigitalfalten, Fingerspitzen und Handrücken

(nach $[6,31]$ ) 
Handekzeme in der COVID-19Pandemie

Im Rahmen der COVID-19-Pandemie kam es durch die neu auferlegten Hygienevorschriften zu einer deutlichen Zunahme der Handhygienemaßnahmen. Die Bundeszentrale für gesundheitliche Aufklärung (BZgA) empfiehlt regelmäßiges Händewaschen mit Seife für mindestens 20-30 s. Auch im Gesundheitswesen wurden Handhygienemaßnahmen mittels Desinfektion und Händewaschen intensiviert. Damit stellt sich die Frage, welche Auswirkung die genannten Maßnahmen auf die Inzidenz von Handekzemen haben. In einer Studie konnte eine Frequenz von Handekzeme bei 50,4\% der Mitarbeiter im Gesundheitswesen, die in die Versorgung von COVID-19-Patienten involviert sind, nachgewiesen werden [36]. In einer anderen Studie aus München berichteten 90,4\% der auf COVID-19-Stationen beschäftigten Mitarbeiter Symptome, die mit einem akuten Handekzem assoziiert sind, wobei ein tatsächliches Handekzem nur bei 14,9\% der Probanden nachgewiesen werden konnte [37].

Merke

Da Hygienemaßnahmen unseren Alltag voraussichtlich noch eine lange Zeit begleiten werden, besteht die Herausforderung darin, der Entstehung von Handekzemen sowohl in der Normalbevölkerung als auch im Gesundheitswesen durch geeignete Präventionsmaßnahmen vorzubeugen.

KERNAUSSAGEN

- Handekzeme gehören zu den häufigen dermatologischen Erkrankungen und haben eine große gesundheitsökonomische Bedeutung.

- Die Genese des Handekzems ist multifaktoriell, eine einheitliche und allgemeingültige Klassifikation existiert bisher nicht.

- Die drei häufigsten Faktoren, die zur Auslösung eines Handekzems führen, sind irritative Schädigung, Kontaktallergie und atopische Diathese.

- Die Therapie des Handekzems erfolgt stadienadaptiert. Oft ist ein multimodales Therapiekonzept erforderlich, das neben Basispflegemaßnahmen, topischer Therapie und UV-Licht auch den Einsatz systemischer Medikamente vorsieht.

- Beruflich verursachte oder verschlimmerte Handekzeme können zur Berufskrankheit (BK-Nr. 5101) führen. Besteht der Verdacht auf ein beruflich getriggertes Handekzem, sollte ein Hautarztverfahren mittels Hautarztbericht eingeleitet werden.
Schlüsselwörter

Kontaktekzem, stadiengerechte Therapie, irritatives Kontaktekzem, Berufsdermatose, atopisches Ekzem

Interessenkonflikt

Erklärung zu finanziellen Interessen

Forschungsförderung erhalten: nein; Honorar/geldwerten Vorteil für Referententätigkeit erhalten: nein; Bezahlter Berater/interner Schulungsreferent/Gehaltsempfänger: nein; Patent/Geschäftsanteile/Aktien (Autor/Partner, Ehepartner, Kinder) an Firma (Nicht-Sponsor der Veranstaltung): nein; Patent/Geschäftsanteile/Aktien (Autor/Partner, Ehepartner, Kinder) an Firma (Sponsor der Veranstaltung): nein.

Erklärung zu nicht-finanziellen Interessen

Die Autorinnen/Autoren geben an, dass kein Interessenkonflikt besteht.

\section{Autorinnen/Autoren}

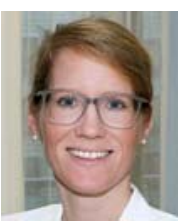

\section{Vera Baur}

Jahrgang 1982; Dr. med., Fachärztin für Dermatologie, Zusatzbezeichnung: Allergologie. 2001-2008 Studium der Humanmedizin an der Philipps-Universität Marburg. 2008-2014 Weiterbildungsassistentin und seit 2015 Oberärztin an der Klinik für Dermatologie, Universitätsklinik der Paracelsus Medizinischen Privatuniversität, Klinikum Nürnberg Nord. Tätigkeitsschwerpunkte: Allergologie, Dermatochirurgie, allgemeine Dermatologie. Klinische Schwerpunkte: Allergologie

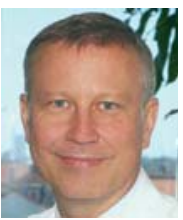

\section{Erwin S. Schultz}

Prof. Dr. med. Studium der Humanmedizin an der Julius-Maximilians-Universität Würzburg. 1998-2000 Forschungsaufenthalt am Ludwig Institute for Cancer Research, Brüssel. 1998 Facharzt für Haut- und Geschlechtskrankheiten. 2005-2008 Professor für Dermatoonkologie, Philipps-Universität, Marburg. 2002 Habilitation. Seit 2008 Leitender Arzt der Klinik für Dermatologie, Universitätsklinik der Paracelsus Medizinischen Privatuniversität, Klinikum Nürnberg Nord.

\section{Korrespondenzadresse}

Dr. med. Vera Baur

Klinik für Dermatologie

Universitätsklinik der Paracelsus Medizinischen

Privatuniversität

Klinikum Nürnberg, Standort Nord

Prof.-Ernst-Nathan-Str. 1

90419 Nürnberg

Deutschland

E-Mail: vera.baur@klinikum-nuernberg.de 
Wissenschaftlich verantwortlich gemäß Zertifizierungsbestimmungen

Wissenschaftlich verantwortlich gemäß Zertifizierungsbestimmungen für diesen Beitrag ist Dr. med. Vera Baur, Nürnberg.

\section{Literatur}

[1] Thyssen JP, Johansen JD, Linneberg A et al. The epidemiology of hand eczema in the general population-prevalence and main findings. Contact Dermatitis 2010; 62: 75-87. doi:10.1111/j.1600-0536.2009.01669.x

[2] Agner T, Elsner P. Hand eczema: epidemiology, prognosis and prevention. J Eur Acad Dermatol Venereol 2020; 34 (Suppl. 01): 4-12. doi:10.1111/jdv.16061

[3] Jakasa I, Thyssen JP, Kezic S. The role of skin barrier in occupational contact dermatitis. Exp Dermatol 2018; 27: 909914. doi:10.1111/exd.13704

[4] Thyssen JP. The association between filaggrin mutations, hand eczema and contact dermatitis: a clear picture is emerging. Br J Dermatol 2012; 167: 1197-1198. doi:10.1111/bjd.12075

[5] Molin S, Merl J, Dietrich KA et al. The hand eczema proteome: imbalance of epidermal barrier proteins. $\mathrm{Br}$ J Dermatol 2015; 172: 994-1001. doi:10.1111/bjd.13418

[6] Diepgen TL, Andersen KE, Chosidow O et al. Leitlinie für die Diagnose, Prävention und Behandlung des Handekzems Kurzversion. J Dtsch Dermatol Ges 2015; 13: 77-85. doi:10.1111/ddg.12510_suppl

[7] Diepgen TL, Elsner P, Fartasch $\mathrm{M}$ et al. Leitlinie Management von Handekzemen. J Dtsch Dermatol Ges 2009; 7 (Suppl. 03): S1-16

[8] Weisshaar E, Eyerich K. Handekzem: auch weiterhin eine diagnostische und therapeutische Herausforderung. Hautarzt 2019; 70: 752-754. doi:10.1007/s00105-019-04477-2

[9] Veien NK. Acute and recurrent vesicular hand dermatitis. Dermatol Clin 2009; 27: 337-353, vii. doi:10.1016/j. det.2009.05.013

[10] Pesonen M, Koskela K, Aalto-Korte K. Contact urticaria and protein contact dermatitis in the Finnish Register of Occupational Diseases in a period of 12 years. Contact Dermatitis 2020; 83: 1-7. doi:10.1111/cod.13547

[11] Diepgen TL, Sauerbrei W, Fartasch M. Development and validation of diagnostic scores for atopic dermatitis incorporating criteria of data quality and practical usefulness. J Clin Epidemiol 1996; 49: 1031-1038. doi:10.1016/08954356 (96)00119-9

[12] Mahler V, Nast A, Bauer A et al. S3-Leitlinie: Durchführung des Epikutantests mit Kontaktallergenen und Arzneimitteln - Kurzfassung Teil 1. J Dtsch Dermatol Ges 2019; 17: 10751093. doi:10.1111/ddg.13956_g

[13] Krohn S, Geier J, Bauer A et al. Qualitätssicherung im BKVerfahren: Epikutantestungen im Hautarztverfahren der gesetzlichen Unfallversicherung. Allergologie 2020; 43: 464-468

[14] Mahler V, Dickel H. Wichtigste Kontaktallergene beim Handekzem. Hautarzt 2019; 70: 778-789. doi:10.1007| s00105-019-04472-7

[15] Oosterhaven JAF, Uter W, Aberer W. ESSCA Working Group. European Surveillance System on Contact Allergies (ESSCA). et al. Contact allergies in relation to body sites in patients with allergic contact dermatitis. Contact Dermatitis 2019; 80: 263-272. doi: $10.1111 / \operatorname{cod} .13192$

[16] Garzorz-Stark N, Krause L, Lauffer F et al. A novel molecular disease classifier for psoriasis and eczema. Exp Dermatol 2016; 25: 767-74. doi:10.1111/exd.13077

[17] Garzorz-Stark N, Eyerich K. Molekulare Diagnostik des Handekzems [Molecular diagnostics of hand eczema]. Hautarzt 2019; 70: 760-765. doi:10.1007/s00105-0194466-9

[18] Elsner P, Agner T. Hand eczema: treatment. J Eur Acad Dermatol Venereol 2020; 34 (Suppl. 01): 13-21. doi:10.1111/ jdv.16062

[19] Wohlrab J, Staubach P, Augustin M et al. S2k-Leitlinie zum Gebrauch von Präparationen zur lokalen Anwendung auf der Haut (Topika). J Dtsch Dermatol Ges 2018; 16: 376-392. doi:10.1111/ddg.13473_g

[20] Empfehlung zur Qualitätssicherung Wirkstoffdossiers für externe dermatologische Rezepturen GD Gesellschaft für Dermopharmazie. Verfügbar unter (Stand 18.06.2021): https://www.gd-online.de/german/veranstalt/ images2013/GD_Wirkstoffdossiers_30.08.2013.pdf

[21] Christoffers WA, Coenraads PJ, Svensson Å et al. Interventions for hand eczema. Cochrane Database Syst Rev 2019; 4: CD004055. doi:10.1002/14651858.CD004055.pub2

[22] Luger T, Loske KD, Elsner P et al. Topische Dermatotherapie mit Glukokortikoiden. Therapeutischer Index. J Dtsch Dermatol Ges 2004; 2: 629-634. doi:10.1046/j.14390353.2004.03626.x

[23] Wollenberg A, Christen-Zäch S, Taieb A et al. European Task Force on Atopic Dermatitis/EADV Eczema Task Force. ETFAD/EADV Eczema task force 2020 position paper on diagnosis and treatment of atopic dermatitis in adults and children. J Eur Acad Dermatol Venereol 2020; 34: 2717 2744. doi:10.1111/jdv. 16892

[24] Ring J, Möhrenschlager M, Henkel V. The US FDA 'black box' warning for topical calcineurin inhibitors: an ongoing controversy. Drug Saf 2008; 31: 185-198. doi:10.2165/ 00002018-200831030-00001

[25] Schultheis K, Messerschmidt A, Ochsendorf F. Topische Therapie von entzündlichen Dermatosen, Juckreiz und Schmerz sowie Hyperhidrose [Topical therapy of inflammatory dermatoses, pruritus and pain, as well as hyperhidrosis]. Hautarzt 2014; 65: 197-206. doi:10.1007/s00105013-2658-2

[26] van den Bogaard EH, Bergboer JG, Vonk-Bergers M et al. Coal tar induces AHR-dependent skin barrier repair in atopic dermatitis. J Clin Invest 2013; 123: 917-927. doi:10.1172/ JCl65642

[27] Ruzicka T, Lynde CW, Jemec GBE et al. Efficacy and safety of oral alitretinoin (9-cis retinoic acid) in patients with severe chronic hand eczema refractory to topical corticosteroids: results of a randomized, double-blind, placebo-controlled, multicentre trial. $\mathrm{Br}$ J Dermatol 2008; 158: 808-817. doi:10.1111/j.1365-2133.2008.08487.x

[28] Oosterhaven JAF, Voorberg AN, Romeijn GLE et al. Effect of dupilumab on hand eczema in patients with atopic dermatitis: An observational study. J Dermatol 2019; 46: 680-685. doi:10.1111/1346-8138.14982

[29] Reich K, Kabashima K, Peris K et al. Efficacy and safety of baricitinib combined with topical corticosteroids for treatment of moderate to severe atopic dermatitis: a randomized clinical trial. JAMA Dermatol 2020; 156: 1333-1343. doi:10.1001/jamadermatol.2020.3260 
[30] Worm M, Bauer A, Elsner P et al. Efficacy and safety of topical delgocitinib in patients with chronic hand eczema: data from a randomized, double-blind, vehicle-controlled phase Ila study. Br J Dermatol 2020; 182: 1103-1110. doi:10.1111/bjd.18469

[31] Agner T, Held E. Skin protection programmes. Contact Dermatitis 2002; 47: 253-256. doi:10.1034/j.16000536.2002.470501.x

[32] Fartasch M, Diepgen TL, Drexler $\mathrm{H}$ et al. S1 1-Leitlinie „Berufliche Hautmittel: Hautschutz, Hautpflege und Hautreinigung " (ICD 10: L23, L24) Kurzversion. J Dtsch Dermatol Ges 2015; 13: 594-606. doi:10.1111/ddg.12617

[33] Ofenloch R, Apfelbacher C, Weisshaar E. Handekzemregister: Hintergründe, Nutzen und Ausblick: Registerdaten in der Forschung zum Handekzem. Hautarzt 2018; 69: 809814. doi:10.1007/s00105-018-4245-z

[34] Krohn S, Drechsel-Schlund C, Römer W et al. BK-Rechtsreform: Rechtsänderungen bei Berufskrankheiten - Auswirkungen auf die dermatologische Praxis. Dermatologie Beruf Umwelt 2020; 4: 145-148. doi:10.5414/DBX00396

[35] Brans R, Skudlik C, Weisshaar E et al. Multicentre cohort study 'Rehabilitation of Occupational Skin Diseases - Optimization and Quality Assurance of Inpatient Management
(ROQ)': results from a 3-year follow-up. Contact Dermatitis 2016; 75: 205-212. doi:10.1111/cod.12614

[36] Erdem Y, Altunay IK, Aksu Çerman A et al. The risk of hand eczema in healthcare workers during the COVID-19 pandemic: Do we need specific attention or prevention strategies? Contact Dermatitis 2020; 83: 422-423. doi:10.1111/ cod.13632

[37] Guertler A, Moellhoff N, Schenck TL et al. Onset of occupational hand eczema among healthcare workers during the SARS-CoV-2 pandemic: Comparing a single surgical site with a COVID-19 intensive care unit. Contact Dermatitis 2020; 83: 108-114. doi:10.1111/cod.13618

Bibliografie

Akt Dermatol 2021; 47: 385-400

DOI 10.1055/a-1106-9108

ISSN $0340-2541$

(c) 2021. Thieme. All rights reserved.

Georg Thieme Verlag KG, Rüdigerstraße 14,

70469 Stuttgart, Germany

Hinweis: Dieser Beitrag wurde gemäß Erratum vom 11.10.2021 geändert. 


\section{Punkte sammeln auf CME.thieme.de}

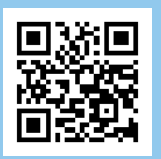

Diese Fortbildungseinheit ist in der Regel 12 Monate online für die Teilnahme verfügbar.

Den genauen Einsendeschluss finden Sie unter https://cme.thieme.de.

Sollten Sie Fragen zur Online-Teilnahme haben, finden Sie unter https://cme.thieme.de/hilfe

eine ausführliche Anleitung. Wir wünschen viel Erfolg beim Beantworten

der Fragen!

Unter https://eref.thieme.de/CXEJNE4 oder über den QR-Code kommen Sie direkt

zur Startseite des Wissenstests.

VNR 2760512021160210062

\section{Frage 1}

Welche Aussage zur Diagnostik bei Handekzemen trifft zu?

A Auf die Durchführung einer mykologischen Diagnostik mittels Pilzkultur kann bei Handekzemen i.d. R. verzichtet werden.

B Bei einem länger als 3 Monate bestehenden Handekzem wird die Durchführung einer Epikutantestung mit der Standardreihe und ggf. berufsspezifischen Reihen empfohlen.

C Mittels Biomarker (NOS2/CCL27) kann das chronische Handekzem von einer Tinea manuum abgegrenzt werden.

D Aufgrund der Epikutantestergebnisse kann auf das Vorliegen einer atopischen Diathese geschlossen werden.

E Handekzeme werden in erster Linie durch die histologische Aufarbeitung einer entnommenen Probebiopsie diagnostiziert.

\section{Frage 2}

Welche Aussage zur Therapie von Handekzemen trifft zu?

A Topische Steroide mit hohem therapeutischem Index $(\mathrm{TIX}=2)$ sollten für die Behandlung von Handekzemen nicht verwendet werden.

B Topische Steroide fördern die Regeneration der Hautbarriere.

C Der Einsatz von Calcineurin-Inhibitoren (Tacrolimus, Pimecrolimus) ist innerhalb der zugelassenen Indikation nur bei Handekzem atopischer Genese möglich.

D Ciclosporin ist für die Behandlung des Handekzems zugelassen.

E Aufgrund der guten Praktikabilität wird meist die BadePUVA- gegenüber der Creme-PUVA-Therapie bevorzugt.

\section{Frage 3}

Welche Aussagen zu beruflichen Einflüssen beim Handekzem trifft zu?

A Das Tragen feuchtigkeitsdichter, okklusiver Schutzhandschuhe ist kein Kriterium für Feuchtarbeit.

B Feuchtarbeitsplätze gehen insgesamt mit einem erhöhten Handekzemrisiko einher.

C Der Hautarztbericht wird durch den behandelnden Arzt erstellt und direkt an den Arbeitgeber des Patienten übermittelt.

D Besteht bei einem Patienten, der sich erstmalig mit einem leichten Handekzem vorstellt, die Möglichkeit, dass berufliche Einflüsse an der Entstehung oder Aggravierung des Handekzems beteiligt sind, sollte der behandelnde Arzt unverzüglich eine Berufskrankheitenanzeige (BK-Anzeige) erstellen

E Handekzeme machen etwa 5-10\% aller Berufskrankheiten aus.

\section{Frage 4}

Ein Handekzem spricht auf eine stadiengerechte, antiekzematöse Handekzemtherapie nicht an. Welche Differenzialdiagnose kann primär unberücksichtigt bleiben?
A Tinea manuum
B Psoriasis vulgaris
C Mycosis fungoides
D systemische Mastozytose
E Lichen ruber 


\section{Punkte sammeln auf CME.thieme.de}

Fortsetzung ...

\section{Frage 5}

Nur eine der folgenden Aussage zum Handekzem trifft zu. Welche?

A Die Lebenszeitprävalenz liegt bei ca. $2 \%$.

B Irritative Handekzeme zeichnen sich vor allem durch eine unscharfe Begrenzung der Hauteffloreszenzen mit Streuherden in der Umgebung aus.

C Handekzeme im akuten Stadium sind v. a. durch das Vorhandensein von Hyperkeratosen und Rhagaden gekennzeichnet.

D Irritative Handekzeme heilen i.d. R. zügig in der arbeitsfreien Zeit ab.

E Faktoren, die zur Auslösung eines Handekzems führen, sind irritative Schädigung, Kontaktallergie und atopische Prädisposition.

\section{Frage 6}

Sie behandeln eine 30-jährige Krankenschwester mit einem seit über 4 Monaten bestehenden Handekzem, welches im Urlaub abheilt. Eine Therapie mit Basispflege und topischen Steroiden während der Arbeitszeit führte zu einer Besserung, jedoch nicht zu einer Abheilung der Ekzeme. Welche Aussage zu dem Fall trifft nicht zu?

A Die Durchführung einer Epikutantestung ist indiziert.

B Ein Hautarztverfahren sollte eingeleitet werden.

C Der Patientin sollte eine sofortige Aufgabe des Berufes und eine Umschulung angeraten werden.

D Unterstützend kann eine Phototherapie (Creme-PUVA) eingeleitet werden.

E Feuchtarbeit (Tragen feuchtigkeitsdichter Handschuhe $>2$ h) kann die Entstehung irritativer Handekzeme begünstigen.

\section{Frage 7}

Nur eine der folgenden Aussagen zur Therapie des chronischen Handekzems trifft zu. Welche?

A Eine Basistherapie mit Rückfettung ist in Therapiestufe I indiziert, in höheren Therapiestufen jedoch nicht mehr erforderlich.

B Eine Leitungswasser-Iontopherese wird i.d. R. über 14 Tage durchgeführt und dann abgesetzt.

C Eine häufige, oft dosisanhängige Nebenwirkung von Alitretinoin sind Kopfschmerzen.

D Ciclosporin sollte idealerweise mit einer Lichttherapie kombiniert werden.

E Methotrexat ist für die Behandlung des hyperkeratotischen Handekzems zugelassen.

\section{Frage 8}

Welche der genannten Substanzen zählt nicht zu den häufigen Kontaktallergenen beim Handekzem?

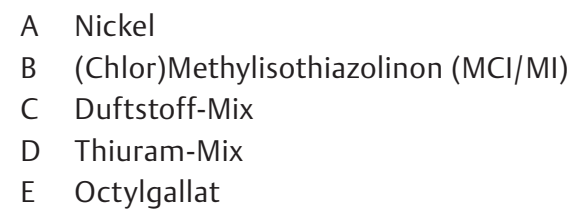

\section{Frage 9}

Eine 40-jähriger Lehrerin leidet unter einem schweren, hyperkeratotisch-rhagadiformen Handekzem idiopathischer Genese. Sie möchten eine Therapie mit Alitretinoin einleiten. Welche Aussage trifft nicht zu?

A Eine Basistherapie mit Hydratation der Haut, Reduktion/ Vermeidung von Triggerfaktoren und Einsatz von Hautschutzmaßnahmen sollte weitergeführt werden.

B Topische Kortikosteroide können zeitgleich eingesetzt werden.

C Eine UV-Therapie ist in Kombination mit Alitretinoin kontraindiziert.

D Die Standarddosierung beträgt $30 \mathrm{mg} / \mathrm{d}$ per os.

E Alitretinoin ist teratogen, deswegen ist der Einschluss in ein Schwangerschaftspräventionsprogramm mit sicherer Kontrazeption erforderlich.

\section{Frage 10}

Was zählt nicht zu den bedeutenden Risikofaktoren für die Entwicklung eines chronischen Handekzems?

A Vorliegen eines atopischen Ekzems in der Eigenanamnese

B großflächiges Handekzem

C Feuchtarbeit

D bekannte Kontaktallergie

E Alkoholkonsum 